\title{
Characteristics, management and response to alteplase in China versus non-China participants of the ENCHANTED trial
}

To cite: Song L, Wang $X$ Robinson T, et al. Characteristics, management and response to alteplase in China versus non-China participants of the ENCHANTED trial. Stroke and Vascular Neurology 2017;2:e000085. doi:10.1136/svn-2017-000085

- Additional material is published online only. To view please visit the journal online (http://dx.doi.org/10.1136/svn2017-000085).

Received 16 March 2017 Revised 29 March 2017 Accepted 2 April 2017 Published Online First 19 May 2017

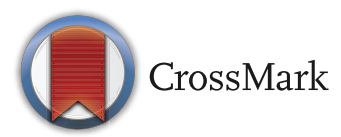

For numbered affiliations see end of article.

Correspondence to Professor Craig S Anderson; canderson@georgeinstitute. org.cn

\section{ABSTRACT}

Background The characteristics of patients with acute ischaemic stroke (AIS) and their management vary across regions, which may influence outcomes. We examined for differential patterns of outcome between China and nonChina participants of the ENhanced Control of Hypertension And Thrombolysis strokE stuDy (ENCHANTED), which tested different alteplase doses in AIS.

Methods ENCHANTED was an international, multicentre, open, blinded-endpoint trial of the effects of low-dose $(0.6 \mathrm{mg} / \mathrm{kg})$ versus standard-dose $(0.9 \mathrm{mg} / \mathrm{kg})$ intravenous alteplase on 90-day disability outcomes and symptomatic intracerebral haemorrhage (sICH) in 3310 patients with AIS. Results Participants $(n=1419,48 \%)$ in China were younger, and more often male, hypertensive and with prior stroke and coronary artery disease, but less likely to have atrial fibrillation and use antihypertensive, antithrombotic and lipid-lowering agents, compared with non-China patients with AIS. Although China participants had more AIS due to large artery occlusion, were treated later and had differing ancillary management, there was no significant difference in 90-day modified Rankin scale scores 2-6 (55.6\% vs $47.8 \%$; 0R, adjusted for baseline and management factors 0.87 (95\% $\mathrm{Cl} 0.71$ to $1.07 ; \mathrm{p}=0.20)$ ) and risk of sICH (Safe Implementation of Thrombolysis in Stroke-Monitoring Study criteria: $1.4 \%$ vs $1.8 \% ; p=0.12$ ) compared with non-China participants. There was no heterogeneity in the treatment effects of low-dose versus standard-dose alteplase between China and non-China participants.

Conclusion Patients with AIS recruited to the ENCHANTED trial in China had similar outcomes in response to thrombolysis treatment despite significantly differing demographic, clinical and management factors to patients with AIS in other regions.

\section{INTRODUCTION}

Stroke is the leading cause of mortality and disability in China. However, Chinese patients with acute ischaemic stroke (AIS) may differ from those in other countries by way of demographic characteristics, ${ }^{1}$ type of vessel occlusion $^{2}$ and in the use of alternative therapies such as traditional Chinese medicine and corticosteroids. ${ }^{3}$ Intravenous alteplase is approved for the treatment of AIS within 4.5 hours of the onset of symptoms, ${ }^{4-6}$ but Chinese patients are often treated later and with lower doses than in the West. ${ }^{7}$

The ENhanced Control of HypertensionAnd Thrombolysis strokE stuDy (ENCHANTED) did not confirm that low-dose alteplase was non-inferior to standard-dose alteplase with respect to the conventional measure of poor outcome, a binary endpoint of death and disability defined by scores of 2-6 on the modified Rankin scale (mRS) at 90 days. However, low-dose alteplase was non-inferior with respect to an ordinal mRS shift analysis and had a significantly lower rate of symptomatic intracerebral haemorrhage $(\mathrm{sICH}) \cdot{ }^{8-10}$ Of the 3310 participants, 1419 were recruited from China, and their demographic and disease profile, management and outcome may have been different from that of patients in other countries. We aimed to quantify differences in characteristics, management and response to treatment by country of recruitment (China vs non-China) in the ENCHANTED trial.

\section{METHODS}

The ENCHANTED trial was an international, multicentre, prospective, randomised, openlabel, blinded-endpoint trial, the details of which are outlined elsewhere. ${ }^{8-10}$ In brief, 3310 patients with AIS confirmed on brain imaging and fulfilling local criteria for thrombolysis treatment, including symptom onset within 4.5 hours, were randomly assigned to receive low-dose $(0.6 \mathrm{mg} / \mathrm{kg} ; 15 \%$ as bolus, $85 \%$ as infusion over 1 hour) or standard-dose $(0.9 \mathrm{mg} / \mathrm{kg} ; 10 \%$ as bolus, $90 \%$ as infusion over 1 hour) intravenous alteplase. The study protocol was approved by the appropriate ethics committee at each participating centre, 
and written informed consent was obtained from each patient or an appropriate surrogate.

Key demographic and clinical characteristics were recorded at the time of enrolment, with neurological severity measured using the National Institutes of Health Stroke Scale and Glasgow Coma Scale at baseline, 24 hours and at day 7 (or earlier on discharge from hospital). The principal clinical outcome was death or level of disability according to the mRS, which was assessed through telephone or in-person contact by researchers who were blind to the randomised treatment allocation at 90 days. Uncompressed digital images of all baseline and follow-up brain scans-CT, MRI and angiograms-were collected in Digital Imaging and Communications in Medicine (DICOM) format on a CD-ROM identified only with the patient's unique study number, and uploaded by a special purpose-built, web-based system for central analysis at The George Institute of Global Health. All brain scans with an intracranial haemorrhage were reviewed by at least two independent assessors blind to clinical data, treatment, and date and sequence of scan using MIStar V.3.2 (Apollo Medical Imaging Technology, Melbourne, Victoria, Australia). Assessors graded any haemorrhage as intracerebral, subarachnoid, intraventricular, subdural or other; sICH was graded across all standard definitions. ${ }^{9}$

Associations of country of recruitment (China vs non-China participants) with death or disability, death and sICH were estimated using logistic regression models with adjustment for confounders. The treatment effects of low-dose versus standard-dose alteplase on death or disability, death and sICH were determined using logistic regression models, and the potential heterogeneity of alteplase dose effect between China and non-China participants was estimated by adding an interaction term to the statistical models. Proportional odds regression models were used to determine the treatment effects on an ordinal shift in mRS scores between China and non-China participants, the proportional odds assumption being fulfilled in all the models. ${ }^{1112}$ Data are reported as ORs and 95\% CIs. Two-sided p values are reported, with $\mathrm{p}<0.05$ considered statistically significant. SAS V.9.3 was used for analyses.

\section{RESULTS}

\section{Baseline characteristics and management}

From March 2012 through August 2015, a total of 3310 patients were randomised; 1654 patients were assigned to low-dose alteplase and 1643 to standard-dose alteplase (after exclusion of 13 patients: 9 had no consent, 1 was mistakenly randomised and 3 had duplicate randomisation). ${ }^{10}$ All 3297 patients (37.8\% female; mean age: 66.6 (SD: 12.8) years) were included in these analyses. Table 1 shows the patient characteristics, split by region, with China participants being younger, and more often male, hypertensive, smokers and with a history of stroke and coronary artery disease, but less likely to have a history of atrial fibrillation and hypercholesterolaemia, and to use antihypertensive, antithrombotic and lipid-lowering agents. Moreover, China participants had a twofold greater risk of large artery occlusion.

Differences in alteplase factors and management over the first 7 days are reported in online supplementary table 1. China participants who received less alteplase, either as a bolus or infusion dose, were treated much later after symptom onset, and were less likely to receive concomitant treatments, including endovascular clot retrieval, neurosurgery, compression stockings, subcutaneous heparin, intubation and ventilation, an antithrombotic, and stroke unit care and rehabilitation. Conversely, they were more likely to receive intravenous blood pressure-lowering treatment, intravenous traditional Chinese medicine and corticosteroids.

Across the whole cohort, independent of randomised treatment, and after adjusting for important baseline variables (model 1) and additionally for early management (model 2), country of recruitment was not associated with death or disability at 90 days, whether defined by mRS scores 2-6 (China vs non-China: OR, 0.87; 95\% CI 0.71 to $1.07 ; \mathrm{p}=0.20)$ or $\mathrm{mRS}$ scores $3-6$ (OR, $1.01 ; 95 \%$ CI 0.81 to $1.26 ; \mathrm{p}=0.93$ ), but was associated with lower mortality (OR, 0.53 ; 95\% CI 0.36 to $0.79 ; \mathrm{p}=0.002$ ) (see online supplementary table 2 ). There was no clear association between country of recruitment and sICH across a broad range of classifications (see online supplementary table 3 ).

When considering the impact of low-dose versus standard-dose alteplase, there was no significant interaction of the treatment effect by country of recruitment for various 90 -day outcomes on the mRS $0-1$ vs $2-6$, or mRS $0-2$ vs $3-6$ and mortality alone, or by ordinal shift analysis (table 2). Low-dose alteplase was associated with less sICH for all patients, with no significant heterogeneity in such risk between China and non-China participants (see online supplementary table 4 ).

\section{DISCUSSION}

In this secondary analysis of the ENCHANTED trial, there were no significant differences in the important outcomes of death or disability, or sICH, between China and non-China thrombolysis-treated patients with AIS, although China participants had lower overall mortality. The treatment effects of low-dose versus standard-dose alteplase on clinical outcomes and sICH were consistent between China and non-China participants.

The median time from the onset of symptoms to receipt of treatment was $200 \mathrm{~min}$ for China participants, which is longer than recorded in the China national stroke registry ${ }^{7}$ and trials or quality assurance studies in other countries. ${ }^{13-15}$ This may have been due to various factors influencing prehospital and posthospital care: delay in the recognition of stroke symptoms, long hospital transfer distances and traffic delays, high cost of alteplase, fear of sICH, and additional efforts required to process the explanation, consent and randomisation aspects of the 
Table 1 Baseline characteristics

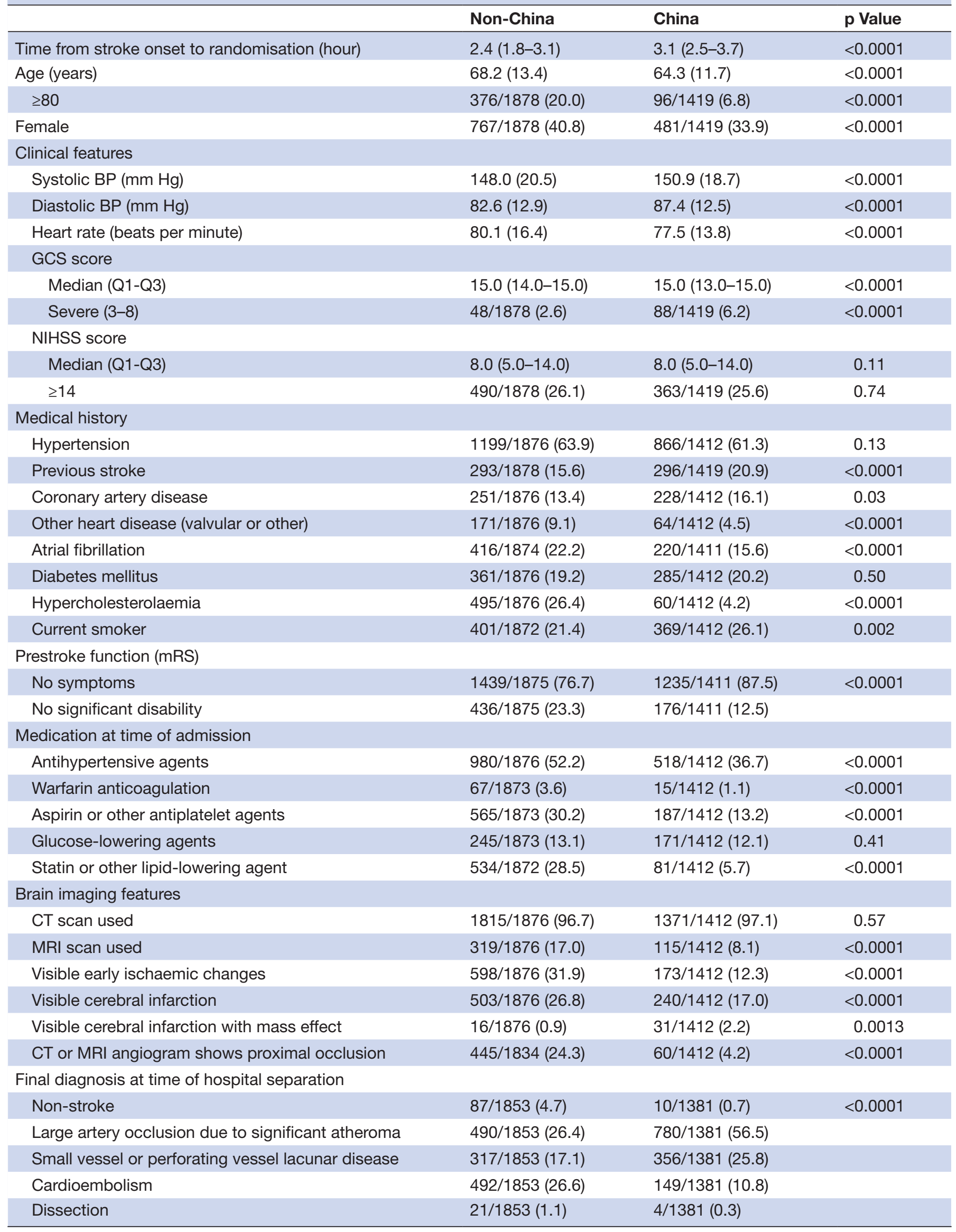


Table 1 Continued

\begin{tabular}{clll}
\hline & Non-China & China & \multicolumn{1}{c}{ p Value } \\
\hline Other or uncertain aetiology & $446 / 1853(24.1)$ & $82 / 1381(5.9)$ & $708 / 1419(49.9)$ \\
Randomised low-dose treatment & $946 / 1878(50.4)$ & 0.79 \\
\hline
\end{tabular}

Data are $\mathrm{n}(\%)$, mean (SD) or median (IQR). $\mathrm{p}$ Values are based on $\chi^{2}$ test, t-test or Wilcoxon signed-rank test.

BP, blood pressure; GCS, Glasgow Coma Scale; mRS, modified Rankin scale; NIHSS, National Institutes of Health Stroke Scale.

trial. China participants appear to have been managed less intensively, for example with regard to investigation (cerebral angiogram), intervention (endovascular clot retrieval, neurosurgery), ancillary care (use of compression stocking and subcutaneous heparin), management (intubation and ventilation, stroke unit care) and hospital rehabilitation.

ENCHANTED was undertaken to resolve uncertainty over the optimal dose of alteplase, as Japanese studies ${ }^{16}$ had indicated low-dose alteplase provides similar, perhaps superior, efficacy and safety to standard-dose alteplase, whereas several observational studies ${ }^{18-23}$ in China had reported conflicting results. Ultimately, as the only randomised evaluation of different doses of alteplase, ENCHANTED failed to confirm that low-dose alteplase was non-inferior to standard-dose alteplase on primary efficacy outcome, but it did clearly indicate that low-dose alteplase was a safer strategy with a lower risk of sICH.

Table 2 Randomised treatment effects on major clinical outcomes at 90 days, by non-China versus China

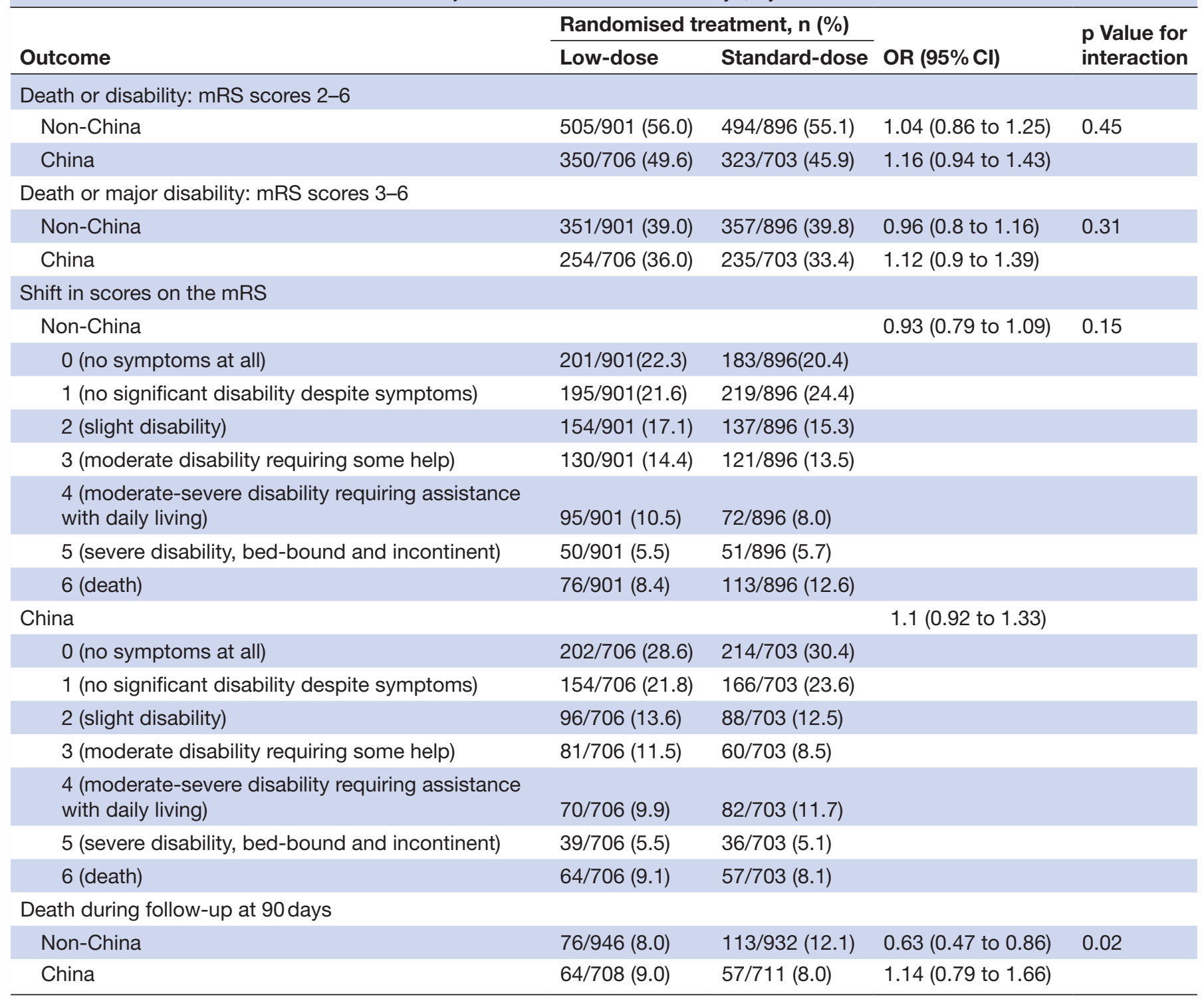

AOR, adjusted OR; mRS, modified Rankin Scale. 
These additional secondary analyses indicate that the efficacy and safety of low-dose versus standard-dose alteplase are consistent between China and non-China participants.

It is difficult to compare our study with other observational studies ${ }^{18-23}$ where there are differences in baseline patient characteristics and stroke severity and use of variable alteplase dose regimens. ${ }^{24} \mathrm{~A}$ multicentre, multinational study ${ }^{25}$ involving 591 patients treated at 48 centres in South Korea, China, India and Singapore reported safety and efficacy of standard-dose alteplase in an Asian population. ${ }^{13}$ Taken together with the current evidence, we consider that standard-dose alteplase should not be excluded as the optimal dose to treat Chinese patients with AIS.

The strengths of this study are the large sample size and inclusion of patients from a variety of healthcare settings, which enhance the generalisability of the results. The high rates of follow-up, adherence to treatment and rigorous assessment of serious adverse events, especially sICH, ensure that the harms were reliably detected and quantified. However, imprecision in the estimates of the treatment effect may have arisen from interobserver variability in mRS scoring in a pragmatic open trial, ${ }^{26}$ while the inclusion of patients with generally milder stroke severity treated with a slightly longer delay from symptom onset than has occurred in other trials and evaluations of alteplase in $\mathrm{AIS}^{14}$ may raise concern over the implications of these results. Finally, this was posthoc subgroup analysis, and consequently imprecision and greater potential random error may have limited our ability to detect significant differences.

In summary, China patients with AIS, as compared with non-China patients with AIS, had different characteristics and management, yet had an increased chance of survival following treatment with alteplase in the ENCHANTED trial. The effects of low-dose versus standard-dose alteplase on all outcomes, including mortality, were similar between China and non-China participants.

\section{Author affiliations}

${ }^{1}$ The George Institute for Global Health at Peking University Health Science Center, Beijing, China

${ }^{2}$ Department of Neurology, 85 Hospital of People's Liberation Army, Shanghai, China ${ }^{3}$ The George Institute for Global Health, The University of New South Wales, Sydney, Australia

${ }^{4}$ Department of Cardiovascular Sciences and NIHR Biomedical Research Unit for Cardiovascular Sciences, University of Leicester, Leicester, UK

${ }^{5}$ Department of Preventive Medicine and Public Health, Faculty of Medicine,

Fukuoka University, Fukuoka, Japan

${ }^{6}$ Clínica Alemana de Santiago, Universidad del Desarrollo, Concepción, Chile ${ }^{7}$ Departamento de Ciencias Neurológicas, Facultad de Medicina, Universidad de Chile, Concepción, Chile

${ }^{8}$ Neurology Department, Royal Prince Alfred Hospital, Sydney, Australia

Contributors CSA contributed to study design, organisation, execution, statistical review, and review and critique of the report. LS contributed to study execution and writing of the report. XW contributed to data analysis and review and critique of the report. TR and RIL contributed to study design, organisation, execution and review and critique of the report. HA and JC contributed to study design and review and critique of the report. PML and XC contributed to study organisation, execution and review and critique of the report.

Funding Funding was principally received from the National Health and Medical Research Council (NHMRC) of Australia. Additional funding was from the Stroke
Association of the United Kingdom, the National Council for Scientific and Technological Development of Brazil, and the Ministry for Health, Welfare and Family Affairs of the Republic of Korea (H114C1985)

Competing interests T Robinson is a National Institute of Health Research Senior Investigator, and reports receiving speaking fees from Bayer and Boehringer Ingelheim, and fees for Advisory Panels from Bayer and Daiichi Sankyo. PM Lavados reports receiving research funding from Astra Zeneca, Bayer and Boehringer Ingelheim and speaking fees from Bayer. RI Lindley reports receiving speaking fees from Boehringer Ingelheim, Covidien, and Pfizer. CS Anderson reports receiving fees for Advisory Panels of Astra Zeneca and Medtronic, speaking at seminars for Takeda China and Boehringer Ingelheim, and a research grant from Takeda China. $\mathrm{J}$ Chalmers reports research grants and lecture fees from Servier for the ADVANCE trial and post-trial follow-up.

Patient consent Obtained.

Ethics approval Every ethics committee at the participating centers.

Provenance and peer review Not commissioned; externally peer reviewed. Data sharing statement No data sharing.

Open Access This is an Open Access article distributed in accordance with the Creative Commons Attribution Non Commercial (CC BY-NC 4.0) license, which permits others to distribute, remix, adapt, build upon this work non-commercially, and license their derivative works on different terms, provided the original work is properly cited and the use is non-commercial. See: http://creativecommons.org/ licenses/by-nc/4.0/

(c) Article author(s) (or their employer(s) unless otherwise stated in the text of the article) 2017. All rights reserved. No commercial use is permitted unless otherwise expressly granted.

\section{REFERENCES}

1. Liu M, Wu B, Wang WZ, et al. Stroke in China: epidemiology, prevention, and management strategies. Lancet Neurol 2007;6:456-64

2. Wang $Y$, Zhao X, Liu L, et al. Prevalence and outcomes of symptomatic intracranial large artery stenoses and occlusions in China: the Chinese Intracranial Atherosclerosis (CICAS) Study. Stroke 2014:45:663-9.

3. Wei JW, Heeley EL, Wang JG, et al. Comparison of recovery patterns and prognostic indicators for ischemic and hemorrhagic stroke in China: the ChinaQUEST (QUality Evaluation of Stroke Care and Treatment) Registry study. Stroke 2010;41:1877-83.

4. Jauch EC, Saver JL, Adams HP, et al. Guidelines for the early management of patients with acute ischemic stroke: a guideline for healthcare professionals from the American Heart Association/ American Stroke Association. Stroke 2013;44:870-947.

5. Demaerschalk BM, Kleindorfer DO, Adeoye OM, et al. Scientific rationale for the inclusion and exclusion criteria for intravenous alteplase in acute ischemic stroke: a statement for healthcare professionals from the American Heart Association/American Stroke Association. Stroke 2016;47:581-641.

6. European Stroke Organisation (ESO) Executive Committee. Guidelines for management of ischaemic stroke and transient ischaemic attack 2008. Cerebrovasc Dis 2008;25:457-507.

7. Wang $Y$, Liao $X$, Zhao $X$, et al. Using recombinant tissue plasminogen activator to treat acute ischemic stroke in China: analysis of the results from the Chinese National Stroke Registry (CNSR). Stroke 2011;42:1658-64.

8. Anderson CS, Woodward M, Arima $\mathrm{H}$, et al. Statistical analysis plan for evaluating low- vs. standard-dose alteplase in the ENhanced Control of Hypertension and Thrombolysis strokE stuDy (ENCHANTED). Int J Stroke 2015;10:1313-5.

9. Huang Y, Sharma VK, Robinson T, et al. Rationale, design, and progress of the ENhanced Control of Hypertension ANd Thrombolysis strokE stuDy (ENCHANTED) trial: an international multicenter $2 \times 2$ quasi-factorial randomized controlled trial of low- vs. standard-dose rt-PA and early intensive vs. guidelinerecommended blood pressure lowering in patients with acute ischaemic stroke eligible for thrombolysis treatment. Int J Stroke 2015;10:778-88.

10. Anderson CS, Robinson T, Lindley RI, et al. Low-dose versus standard-dose intravenous alteplase in acute ischemic stroke. $N$ Engl $J$ Med 2016;374:2313-23.

11. Woodward M. Epidemiology Study Design and Data Analysis. America: Chapman \& Hall/CRC, 2013.

12. Bath $P M$, Lees $K R$, Schellinger $P D$, et al. Statistical analysis of the primary outcome in acute stroke trials. Stroke 2012;43:1171-8. 
13. Wahlgren N, Ahmed N, Dávalos A, et al. Thrombolysis with alteplase for acute ischaemic stroke in the Safe Implementation of Thrombolysis in Stroke-Monitoring Study (SITS-MOST): an observational study. Lancet 2007;369:275-82.

14. Emberson J, Lees KR, Lyden P, et al. Effect of treatment delay, age, and stroke severity on the effects of intravenous thrombolysis with alteplase for acute ischaemic stroke: a meta-analysis of individual patient data from randomised trials. Lancet 2014;384:1929-35.

15. Tissue plasminogen activator for acute ischemic stroke. the National Institute of neurological disorders and Stroke rt-PA Stroke Study Group. N Engl J Med 1995;333:1581-7.

16. Mori E, Minematsu K, Nakagawara J, et al. Effects of $0.6 \mathrm{mg} / \mathrm{kg}$ intravenous alteplase on vascular and clinical outcomes in middle cerebral artery occlusion: Japan Alteplase Clinical Trial II (J-ACT II). Stroke 2010;41:461-5.

17. Yamaguchi T, Mori E, Minematsu K, et al. Alteplase at $0.6 \mathrm{mg} / \mathrm{kg}$ for acute ischemic stroke within 3 hours of onset: Japan Alteplase Clinical Trial (J-ACT). Stroke 2006;37:1810-5.

18. Chao AC, Hsu HY, Chung CP, et al. Outcomes of thrombolytic therapy for acute ischemic stroke in Chinese patients: the Taiwan Thrombolytic Therapy for Acute Ischemic Stroke (TTT-AIS) study. Stroke 2010;41:885-90.

19. Chao AC, Liu CK, Chen $\mathrm{CH}$, et al. Different doses of recombinant tissue-type plasminogen activator for acute stroke in Chinese patients. Stroke 2014;45:2359-65.
20. Chen $\mathrm{CH}$, Hsieh CY, Lai TB, et al. Optimal dose for stroke thrombolysis in Asians: low dose may have similar safety and efficacy as standard dose. J Thromb Haemost 2012;10:1270-5.

21. Liao X, Wang $\mathrm{Y}$, Pan $\mathrm{Y}$, et al. Standard-dose intravenous tissue-type plasminogen activator for stroke is better than low doses. Stroke 2014;45:2354-8.

22. Pan SM, Liu JF, Liu M, et al. Efficacy and safety of a modified intravenous recombinant tissue plasminogen activator regimen in Chinese patients with acute ischemic stroke. J Stroke Cerebrovasc Dis 2013;22:690-3.

23. Zhou XY, Wang SS, Collins ML, et al. Efficacy and safety of different doses of intravenous tissue plasminogen activator in Chinese patients with ischemic stroke. J Clin Neurosci 2010;17:988-92.

24. Sharma VK, Ng KW, Venketasubramanian N, et al. Current status of intravenous thrombolysis for acute ischemic stroke in Asia. Int $J$ Stroke 2011;6:523-30.

25. Rha JH, Shrivastava VP, Wang Y, et al. Thrombolysis for acute ischaemic stroke with alteplase in an Asian population: results of the multicenter, multinational Safe Implementation of Thrombolysis in Stroke-Non-European Union World (SITS-NEW). Int J Stroke 2014;9:93-101.

26. Quinn TJ, Dawson J, Walters MR, et al. Reliability of the modified Rankin Scale: a systematic review. Stroke 2009;40:3393-5. 